G. Dr. J. showed it to the Society for Medical Improvement, and he and Dr. Coolidge gave subsequently the following description :-

"The mass was of an oval form ; $8 \frac{1}{4}$ inches in length, $6 \frac{1}{4}$ inches in width, and 5 inches in thickness; weight, 5 lbs. $3 \frac{1}{2}$ oz. Upon one surface there were the remains of the kidney, about two inches in extent; and, including this, was a somewhat defined, oval, superficial patch, about $6 \frac{1}{2}$ inches by $2 \frac{1}{2}$ inches in extent, and that strongly sug. gested the idea of a connection with the original outline of the organ. Around this patch the surface was lobulated, and radiated in appearance, but upon the opposite surface it was much more even. The irregularity was much more apparent after a fibrous envelop had been stripped off that was quite marked for the most part, and moderately adherent by a lax tissue, though less so to the patch above referred to than to some other parts. Towards the surface where the patch was, and throughout nearly one half of the whole mass, the structure was white, not vascular, had a pearly translucency, and was softer than fœtal brain, with an appearance as if it were infiltrated with a ropy fluid. The remainder was much firmer, and evidently contained more or less connective tissue, the softer and firmer portions passing gradually into each other. This last was also stained by blood, and in some parts quite deeply, though no free clots were found. There was also a small serous cyst in this firmer portion. The portion of kidney above referred to was perfectly healthy in appearance; and, on incision, there was found, within, a well-marked pelvis that could be traced for three or four inches, and infundibula arising from it, with portions of the renal substance that had undergoue more or less the cancerous transformation. There were also found in distant parts, but upon the surface of the mass only, several small, insulated remaius of perfectly healthy renal substance, about three or four lines in diameter.

Under the microscope, the soft substance which constituted so large a part of the disease consisted of innumerable, roundish cells, not varying much in size or form. They had large nuclei, and were embedded in a soft, streaky, ropy mass. At the end of a week, the specimen not having been preserved in any way, the nuclei alone were visible; the substance of the cells having become undistinguishably fused with the mass in which they were."

\section{TEDIOUS LABOR; INCISION OF OS AND CERVIX UTERI.}

By Johx L. ScllivaN, M.D., Malden.

Mrs. F. D., aged 25, primipara, well formed, muscular. Labor pains first felt on the evening of 10th inst. When examined, at 9, P.M., of the 11 th inst., os was found too small to admit the point of the fore-finger; presentation probably cephalic ; pains light, but regular, recurring at intervals of fifteen minutes; passages moist, cool ; perinæum relaxed.

12th, 9, A.M.-Pains all night, preventing sleep; os unchanged. During the day slight, bloody show ; membranes ruptured; waters discharged.

11, P.M._-Os dilated so as barely to admit fore-finger; head presenting; pains every five minutes, and severer; patient fatigued and somewhat desponding. Opium (grs. iij.) administered.

13th, 9, A.M.-Slept "by snatches" during night; pains every three or five minutes; slight thirst; no appetite. Warm hip bath, enema per rectum, tartar emetic ad nauseam ; warm vaginal douche proposed, but objected to.

10, P.M.-Pains increasing; head descending through pelvis, driving uterine neck before it; no farther dilatation; os very rigid. With difficulty, a No. 1 Barnes's dilator introduced and inflated. Pains very severe; dilator removed after an hour. Os dilated to about the size of half a dollar, still rigid; presentation readily diagnosticated r. o. c. Largest sized dilator introduced, followed by very frequent and severe uterine contractions. Dilator removed in half an hour. Os two inches in diameter ; edge like whip-cord.

14th, 1, A.M.-Dilator introduced and fully expanded. Pains violent and almost uninterrupted.

$1 \frac{1}{2}$, A.M.-Dilator remored. No permamanent increase in dilatation of os, which seemed to contract like India-rubber on removal of dilator. Patient's condition still good; passages moist; tongue ditto; abdomen not tender; uterine contractions regular and quite forcible. Determined to leave the case to nature until symptoms of constitutional irritation should supervene, rendering farther assistance indispensable to the safety of mother or child, or of both. 10, P.M.-No progress towards delivery. $O s$ rigid and undilatable as ever; uterine neck swollen and odematuus, pitting deeply on pressure; pains frequent, powerful and agonizing; passages dry and hot; abdomen tender on pressure; tongue dry and 
burning; thirst urgent; pulse frequent and hard. One ounce of dark-looking urine drawn by catheter.

It was clear that the safety of mother and child depended upon speedy delivery, and that this could not be eflected without assistance. Forceps or incision of the os uteri were the operative measures which suggested themselves. In view of the difficulty of drawing the head through the swollen and unyielding cervix, and the fatal conse. quences that might ensue from failure in the atternpt, the latter alternative, incision of the os, was chosen. The blade of a hernia knife was carefully introduced between the fotal head and the cervix uteri, in the interval between the pains. As soon as the uterine contraction occurred, an incision was made upon the anterior lip through the os and cervix, to the depth of about an inch and a half, care being taken to carry the division of the uterine fibres deep enough to insure the ready passage of the head, and thus obviate in some degree the danger of laceration. This procedure was followed by a discharge of some fluid blood and several large coagula, which relieved the œdematous condition of the neck. Several short and hesitating pains succeeded, as if the womb, startled by some unusual occurrence, had paused to reconnoitre; then came a prolonged, expulsive effort, and the child's head passed fairly through the os, distending the perinæum.

The termination of the labor was easily accomplished, and in less than half an hour a full-term male child was delivered. Unfortunately it had cyanosis, and in consequence survived only twenty-four hours. The mother experienced no, untoward syrnptoms, either from the tediousness of the labor or the means employed for her relief, and is now (March 31st) convalescent. Duration of her labor, 102 hours.

In a few minutes after the birth of the child, the uterus, aided by very moderate pressure over the fundus, contracted firmJy, expelling the placenta. The binder having been applied, a vaginal examination was made, to ascertain the condition of the cervix. A shallow notch marked the place where the incision had been made. There was no subsequent hæmorrhage, the lochia being even less profuse than usual.

With reference to the foregoing operation, Dr. Murphy, in his Lectures on the Principles and Practice of Midwifery (Second Edition), pages 246 and 247 , observes : "If these means fail, it becomes a question whether we should wait for the death of the child, in order to remove it by the crotchet, or incise the unyielding cervix. The former practice involves a sacrifice of life, but generally secures the mother from the injurious effects which may follow. The latter may be the means of preserving the child; but if the incision lead to a laceration of the uterus, the mother is at once placed in imminent danger of her life. The fear of such a consequence, it appears to us, has prevented any attempt from being made thus to cut through this Gordian knot of difficult labor in its first stage; but whether this, like other operations, is only surrounded by chimeras of the imagination, which some bold spirit will dissipate, remains yet to be proved. Incision has been performed without accident; the same may happen again, and we confess, in a case such as we have described to you, we should be more disposed to adopt the shorter course, in the hope of saving the child, than to wait until its death enabled us to remove it. This, however, is but an individual opinion, and needs support." .

He adds the following in a foot-note:"Since these observations were first written (1845), several cases have been recorded in which the cervix has been incised, the child saved, and no mother lost. Vide Mr. Tweddle's case (Guy's Hospital Reports, vol. iv. p. 119) ; Mr. Butler's (Medical Gazette, vol. xx. p. 589); Dr. Buckminster's (American Quarterly Journal, October, 1847) ; Dr. Pagan's (Edinburgh Monthly Journal, August, 1854, p. 172)."

He refrains, however, from commenting upon these casese expressing no opinion whether in his judgment incision of the cervix uteri should be included in the category of legitimate operations.

"Multiple incisions upon the neck" are recommended by Cazeaux, who asserts that " they render consecutive laceration far less probable than would a single incision." He adds :- "Very rarely will it be necessary to make them more than half an inch long, while they may be often less than this ; for it is the almost universal practice to be content with cuts of from three to four sixteenths of an inch in depth only, around the circumference of the orifice. The lateral parts of the neck should be chosen for the incision, though, if necessary, they may be made upon the anterior lip, and, lastly, upon the posterior lip."

While not presuming to place our humble opinion in opposition to that of the distinguished obstetricians just quoted, we must confess that we are at loss to perceive the advantage of "multiple incisions" when one will prove equally safe and effi- 
cient. It seems to us that, when practicable, a single incision should be made sufficiently free to ensure the easy escape of the head. If this be done, it is difficult to conceive why a consecutive laceration should occur. Indeed, both experience and reflection have.convinced us that, when the incision is properly made, the danger of such an accident must be very small, if not purely hypothetical.

On the other hand, if the incision be not carried deep enough to accomplish per se the desired object, viz., the liberation of the head, it is difficult to understand why laceration should not take place, especially if the subsequent pains be violent and prolonged, as they naturally would be, excepting in cases of exhaustion, either general or of the uterus alone.

If the uterus were contracting vigorously, a laceration of greater or less extent would be very likely to ensue, when the obstacle to the advance of the head had been only partially removed by a timid and inefficient use of the knife. It is easy to comprehend how a slight cut or nick in the cervix might weaken its power of resistance at that spot, and thus become the point of departure of a dangerous, if not fatal laceration. This is certain-the depth to which an incision is carried is wholly within the operator's control; the extent to which laceration may take place lies wholly beyond it.

It seems to us, therefore, that if the knife is to be used at all, it should be used freely, so that when the uterus arain contracts forcibly it may meet with little or no resistance from its own tissues to the descent of the fotal head. Should it appear that a sufficiently free incision could not be made through one lip only without endangering the integrity of the intra-uterine or of the abdominal structures, we are aware of no anatomical nor surgical reason forbidding the division of both the anterior and posterior lips, either at corresponding points of their respective segments, or at whatever point in each the greatest impediment seems to exist.

Such is the method, founded, as it appears to us, on ratioual and obvious principles, which we would submit for the guidance of the practitioner, or commend, at least, to his attentive consideration whenever it is proposed, in the language of Dr. Murphy, "to cut through this Gordian knot of difficult labor."

In the case we have detailed, we are persuaded that had we waited for " the death of the child in order to remove it by the crotchet," the mother would probably have perished also. Nearly equal danger to both would have attended the use of the forceps; and, had these failed, there would have remained no alternative but craniotomy, performed under circumstances highly unfavorable to the mother.

It remains to notice the possibility of dangerous hæmorrhage occurring as a consequence of the free incisions we have recommended, let it be understood, in exceptional cases only. We can conceive that such an accident might result, ist, as a very rare coincidence, from the patient's possessing the hæmorrhagic diathesis or idiosyncrasy. 2d. Inertia uteri, spontaneous, or following anæsthesia from ether or chloroform, might give rise to flooding dangerous in itself, and rendered more so by the additional loss of blood to which incisions under these circumstances might give rise. As a precaution, therefore, just before the operation is performed a full dose of ergot might with propriety be administered, unless the patient were a primipara, or the medicine otherwsie contra-indicated. And even in a first labor, should the uterine contractions have become feeble and infrequent, or have ceased altogether, and an evident necessity existed for stimulating the womb, ergot in small and tentative doses might be resorted to. It should be borne in mind, however, that ergot in such a case would be inadmissible unless it were evident that delivery could be effected before there had been time for its absorption. An attentive examination of the perinæum and external parts will enable the experienced practitioner to determine with sufficient accuracy the probable duration of this stage of the labor. If ergot is contra-indicated, opium, given in small and repeated doses, will prove a uterine motorstimulant, to the good effects of which we can testify from long experience. Small as the danger probably is of any considerable hæmorrhage arising from incision of the cervix, our own judgment would be decidedly against the performance of this operation unless the uterus were contracting regularly and with power sufficient effectually to close the divided vessels.

New Editors of the St. Louis Medical, Reporter.-Drs. W. M. McPheeters and G. M. B. Maughs succeed Dr. O. F. Potter in the editorial management of the St. Louis Medical Reporter. In future this Journal will be published monthly, instead of twice a month, as in the past. $-N$. Y. Med. Rec. 